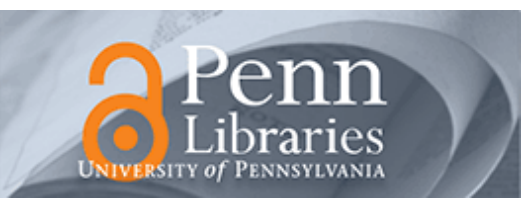

University of Pennsylvania

ScholarlyCommons

March 2003

\title{
HIV Viral Docking: Model Predictions for Bond Number and Trajectory
}

\author{
Thomas J. English \\ University of Pennsylvania \\ Daniel A. Hammer \\ University of Pennsylvania, hammer@seas.upenn.edu
}

Follow this and additional works at: https://repository.upenn.edu/be_papers

\section{Recommended Citation}

English, T. J., \& Hammer, D. A. (2003). HIV Viral Docking: Model Predictions for Bond Number and Trajectory. Retrieved from https://repository.upenn.edu/be_papers/32

Copyright 2003 IEEE. Reprinted from Proceedings of the 29th IEEE Annual Bioengineering Conference 2003, pages 156-157.

Publisher URL: http://ieeexplore.ieee.org/xpl/tocresult.jsp?isNumber=27351\&page=5

This material is posted here with permission of the IEEE. Such permission of the IEEE does not in any way imply IEEE endorsement of any of the University of Pennsylvania's products or services. Internal or personal use of this material is permitted. However, permission to reprint/republish this material for advertising or promotional purposes or for creating new collective works for resale or redistribution must be obtained from the IEEE by writing to pubs-permissions@ieee.org. By choosing to view this document, you agree to all provisions of the copyright laws protecting it.

This paper is posted at ScholarlyCommons. https://repository.upenn.edu/be_papers/32

For more information, please contact repository@pobox.upenn.edu. 


\title{
HIV Viral Docking: Model Predictions for Bond Number and Trajectory
}

\begin{abstract}
Viruses are nano-scale pathogenic particles. Understanding viral attachment is important to understand infectivity, disease transmission, and virus propagation throughout the host. A new simulation technique has been developed to study viral docking behavior - Brownian Adhesive Dynamics (BRAD). BRAD couples Brownian motion algorithm with adhesive dynamic models, and incorporates the effect of virus/cell geometry - an improvement over previous models. The method is extendable to any virus/cell system as well as nanoparticle adhesion system. Current studies have focused on the HIV/CD4 cell system. Comparison of BRAD simulation predictions with those of previous models of viral ducking has shown differences in steady state bond number and bond trajectory. This indicates that geometry of the system plays a significant role in the bonding behavior of viruses. Thus, it is shown that the equivalent site hypothesis is suspect.

Comments

Copyright 2003 IEEE. Reprinted from Proceedings of the 29th IEEE Annual Bioengineering Conference 2003, pages 156-157.

Publisher URL: http://ieeexplore.ieee.org/xpl/tocresult.jsp?isNumber=27351\&page=5

This material is posted here with permission of the IEEE. Such permission of the IEEE does not in any way imply IEEE endorsement of any of the University of Pennsylvania's products or services. Internal or personal use of this material is permitted. However, permission to reprint/republish this material for advertising or promotional purposes or for creating new collective works for resale or redistribution must be obtained from the IEEE by writing to pubs-permissions@ieee.org. By choosing to view this document, you agree to all provisions of the copyright laws protecting it.
\end{abstract}




\section{HIV Viral Docking: Model Predictions for Bond Number and Trajectory}

Thomas J. English and Daniel A. Hammer

Department of Chemical and Biomolecular Engineering, University of Pennsylvania, Philadelphia, PA 19104, USA

\begin{abstract}
Viruses are nano-scale pathogenic particles. Understanding viral attachment is important to understand infectivity, disease transmission, and virus propagation throughout the host. A new simulation technique has been developed to study viral docking behavior - Brownian Adhesive Dynamics (BRAD). BRAD couples Brownian motion algorithms with adhesive dynamic models, and incorporates the effect of virus/cell geometry - an improvement over previous models. The method is extendable to any virus/cell system as well as nanoparticle adhesion systems. Current studies have focused on the HIV/CD4 cell system. Comparison of BRAD simulation predictions with those of previous models of viral docking has shown differences in steady state bond number and bond trajectory. This indicates that geometry of the system plays a significant role in the bonding behavior of viruses. Thus, it is shown that the equivalent site hypothesis is suspect.
\end{abstract}

\section{INTRODUCTION}

A simplified view of a virus life cycle has four steps. First, the virus attaches to a host cell. HIV uses the viral glycoprotein gp120 on its envelope to attach to the CD4 protein on cells. Second, the viral genetic material is inserted into the host cell - in the case of HIV this is done through endocytosis. Third, the viral genetic material takes over the operation of the host cell, forcing the host cell to manufacture new virus. Fourth, the cell lyses, releasing newly created virus that repeat the cycle [1]. Given the severity of HIV/AIDS, accurate quantitative models of the host specificity and binding of HIV are essential. Accurate models of viral docking will provide insight into how viruses partition into different cell types, how infection propagates, and how to prevent transmission.

Currently there is one dominant model of viral docking. Mass balances are written for the population of virus bound by $i$ receptors. These balances include rate terms that account for a virus forming or breaking bonds. Thus, the model consists of a set of $n$ differential equations, corresponding to the maximum number of bonds. There is also an algebraic constraint within the model based upon the fact that the sum of potential binding sites and bound sites on the surface of the cell must sum to the total number of binding sites on the surface of the cell [2-4]. By making the approximation that the number of available cellular binding sites is equal to the total number of binding sites, the model is converted to a set of linear ordinary differential equations. In the linear form the model becomes a continuous time Markov chain. Thus, expected times for dissociation can be calculated. Implicit in this model is the assumption that all binding sites have the same rate of bond formation and breakage. This is known as the equivalent site hypothesis (ESH). However, we postulate that the geometry. of the virus, the distribution and length of viral attachment proteins and cell receptors, and the random forces placed on molecules due to fluctuations in Brownian motion mean that the rates of bond formation and breakage are likely different for each receptor viral attachment protein pair. Hence we suspect the ESH is not strictly valid, and a more detailed method is necessary to accurately simulate viral docking.

Adhesive dynamic simulations are another method of modeling biological attachment [5-7]. The method employs a combination of deterministic equations of motion and probabilistic bond formation and breakage. Adhesive dynamic simulations have had success in prediction and replication of experimental results for adhesion of cells and beads on surfaces [5-7]. The adhesive dynamic method is extendable to any sized particle that undergoes adhesion.

Viruses are nano-sized particles, whose dominant source of motion is derived from the thermal motion of the surrounding solution - i.e., the Peclet number is small. Combining Brownian motion with adhesive dynamic models creates a technique to simulate viral attachment to surfaces. This paper presents Brownian Adhesive Dynamics (BRAD), a new technique capable of modeling viral docking behavior.

\section{METHODS}

Adhesive dynamics is a method that combines deterministic equations of motion with random generation of bond formation and breakage. The velocity of the particle at each time step results from a balance of forces acting on the particle. In the current versions of the method, the forces result from hydrodynamic and colloidal bonding forces. Bonds are modeled as Hookean springs, and are formed and broken according to the Dembo model:

$$
\begin{gathered}
k_{f}=k_{f}^{o} \exp \left(-\frac{\sigma_{t s}\left(x_{m}-\lambda\right)^{2}}{2 K_{b} T}\right) \\
k_{r}=k_{r}^{o} \exp \left(\frac{\left(\sigma-\sigma_{t s}\right)\left(x_{m}-\lambda\right)^{2}}{2 K_{b} T}\right)
\end{gathered}
$$

where $k_{f}$ is the rate of bond formation, $k_{f}^{o}$ is the reference rate of formation, $k_{r}$ is the rate of bond breakage, $k_{r}^{o}$ is the reference rate of breakage, $\sigma$ is the bond spring constant, $\sigma_{t s}$ is the transition "state spring constant, $\lambda$ is the bond's equilibrium length, and $x_{m}$ is the length of the bond. The resulting rates are the rates of an exponential distribution. At each time step bonds are generated to be consistent with the exponential probability distribution given by the Dembo model. Then the forces resulting from these bonds as well as the hydrodynamic forces resulting from an imposed fluid flow and electrostatic repulsive forces are evaluated. With the force acting on the particle known, the equations of motion are integrated up to the next time step. To extend the model to viruses, random forces on the particle resulting from thermal motion are incorporated into the method.

The random motion will consist of translational as well as rotational random walks. Using equations from Allen and 
Tildesley with Newtonian mechanics the equations of motion for the virus particle can be expressed as:

$$
\begin{gathered}
\underline{\dot{p}}(t)=-\xi \underline{p}(t)+\underline{f}(t)+\underline{\circ}(t) \\
\xi=\frac{K_{b} T}{m D}=\frac{K_{b} T}{m} \frac{f_{\text {dag }}}{K_{b} T}=\frac{f_{\text {dag }}}{\text { inertia }}[=] \frac{1}{\text { time }}
\end{gathered}
$$

where an underscore indicates a vector quantity, a solid dot over a variable represents the time derivative of that variable, an open dot over a variable represents a random variable chosen from a particular distribution, $p$ is the momentum of the particle, $f$ is the sum of the applied forces on the particle. The random momentum changes are selected from a Gaussian distribution of possible momentum changes that would be consistent with Boltzman's distribution of molecular energies. The quantity $\xi$ is the viscous relaxation time constant, and physically represents the time for changes in velocities due to molecular collisions to subside. For the HIV system $1 / \xi$ is on the order of $10^{-10}$ seconds. Typical time constants in adhesion dynamic simulations are on the order of $10^{-7}$ seconds. Thus, the momentum equations can be written as equations for position and velocity assuming that the acceleration dynamics have reached steady state and integrated in an Euler fashion using a time step greater than the viscous relaxation time yet smaller than the adhesion dynamic time constant:

$$
\begin{gathered}
\underline{r}(t+\delta)=\underline{r}(t)+c_{1} \delta \underline{v} \underline{(t)}+c_{2} \delta^{2} \underline{a}(t)+\delta \underline{r}^{G} \\
\underline{v}(t+\delta)=c_{0} \underline{v}(t)+\left(c_{1}-c_{2}\right) \delta \underline{\delta} \underline{a}(t)+c_{2} \delta \underline{a}(t+\delta)+\delta \underline{v}^{G}
\end{gathered}
$$

where $r$ is the position vector, $v$ is the velocity vector, $a$ is the acceleration vector, $\delta t$ is the time step, $\delta r^{G}$ is a random position, $\delta v^{G}$ is a random velocity, and $c_{1,2,3}$ are constants resulting from the discretization and conversion from the momentum state equation to the position and velocity state equations. The random positions and velocities are chosen from a bi-variate Gaussian distribution that is derived from the original distribution of momentums [8].

\section{RESULTS}

ESH models and BRAD simulations predictions were compared. All parameters, such as intrinsic rate constants and protein densities, in both models were set using available experimental data. Three examples of differences between the predictions of ESH models and BRAD simulations will be cited here. First, ESH models predict that every virus cell collision results in bond formation while BRAD simulations show that it is possible for a virus to collide with the cell and not form a bond - this occurred for 8 viruses out of 45 viruses simulated. Second, ESH models predict that every virus will reach a steady state of 14 bonds with the cell, while BRAD simulations show that there will be a distribution for the number of steady state bonds (Fig. 1). Third, ESH models predict a smooth monotonic increase in bond number up to the steady state bond number while $\mathrm{BRAD}$ simulations show that bonds can form break and form again en route to the steady state bond number (Fig. 2).

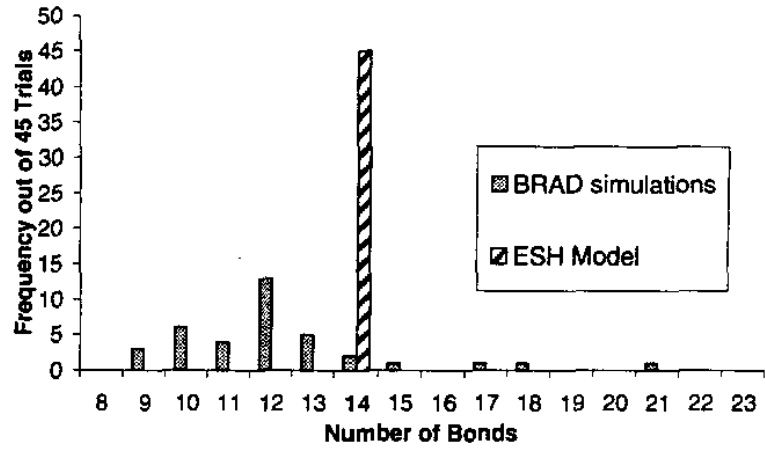

Fig. 1. Comparison of ESH model and BRAD predictions for steady state bond number, for $\mathrm{gp}-120$ mediated HIV docking on CD4 cells.

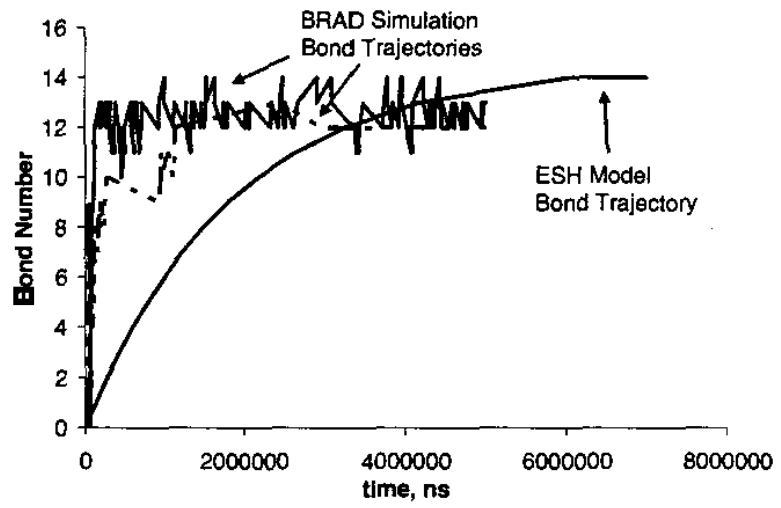

Fig. 2. Comparison of ESH model and BRAD predictions for bond number trajectory, for gp-120 mediated HIV docking on CD4 cells.

\section{DISCUSSION}

BRAD simulations are a powerful new tool to determine interactions between viruses and cells. They are capable of providing information on the rate of bond formation and breakage, as well as the maximum number of bonds between a virus and a cell. BRAD simulations make several improvements over the traditional ESH model of viral docking. These improvements include accounting for the geometry of the virus and cell as well as the positions of the adhesive molecules, independence from the type of distribution from which the rates of bond formation and breakage come, the ability to account for more than one set of adhesion molecules, and the elimination of the equivalent site hypothesis. It is worth noting that the BRAD simulations reach steady state faster than ESH models, even though the same intrinsic rate constants are used for both models. This illustrates the importance of the virus/cell geometry on the dynamics of the system.

[1] A. J. Levine, Viruses (1992)

\section{REFERENCES}

[2] W. S. Hlavacek et al., Proc. Natl. Acad. Sci, vol. 96, 14681 (1999)

[3] A. S. Perelson, Math. Biosci., vol. 53, 1 (1981).

[4] T. J. Wickham et al., Biophys. J., vol. 58, 1501 (1990).

15] D. A. Hammer, S. M. Apte, Biophvs. J., vol. 62, 35 (1992).

16] D. J. Tees et al., J. Chem. Phys., vol. 114, 7483 (2001)

[7] M. R. King, D. A. Hammer, Biophys. J., vol. 81, 799 (2001).

[8] M. P. Allen, D. J. Tildesley, Computer Simulations of Liquids (1987). 